\title{
RENART, PERSONNAGE DES FRONTIÈRES
}

\author{
Yao Lambert KONAN \\ Université Alassane Ouattara (Côte d'Ivoire)
}

\begin{abstract}
En): This study focuses on Reynard, the major character of all aspects of Reynard romance as an elusive and unstable hero, on the edge and always ready to lead astray its enemies as well as the members of the audience or readers. Accepting a changing and rippling identity, fluctuating between cunningness and satanism. This character is at the same time changeability and boundary (combines animal and human traces) and it symbolizes the human struggle between the Good and the Evil.
\end{abstract}

Key-words: satanisme; boundary; human; renardy; Reynard character.

Résumé (fr) : L'étude montre Renart, personnage de tous les univers de la geste renardienne, comme un héros insaisissable et instable à la lisière de tous les espaces, toujours prêt à égarer, aussi bien ses ennemis que le lecteur /spectateur. Déclinant une identité fluctuante et ondoyante, oscillant entre renardie et diabolie, ce personnage à la fois mutant et des frontières (isotopies animale et humaine) symbolise l'Homme aux prises avec les forces du Bien et du Mal.

Mots clés : diabolie, frontière, Homme, renardie, Renart personnage.

\section{Introduction}

L'âge classique médiéval ${ }^{1}$ français a vu naître, dans l'univers des récits drolatiques $^{2}$, un personnage atypique aux exploits déroutants, mais captivants : Renart le goupil ${ }^{3}$. Héros sybillin, caractérisé par la fuite, le personnage donne une cadence actantielle effrénée à la narration fondée sur l'éternel retour et l'immortalité4.

\footnotetext{
${ }^{1}$ Le Classicisme médiéval couvre les XIIe et XIIIe siècles. Ces périodes correspondent à la prospérité agricole, au développement des villes, des grandes réalisations culturelles et artistiques, avec l'ouverture des Universités, notamment celle de Paris en 1200. Elle reçoit, en 1252, le nom de Sorbonne qu'elle porte encore aujourd'hui. Les XIIe et XIIIe siècles sont ainsi qualifiés de Siècles de Lumière, appelés Âge classique médiéval. C'est «le beau Moyen Âge», ou le «Moyen Âge flamboyant », cf : Michel Stanesco, Lire le Moyen Âge, Paris, Dunod, 1998, p. 143.

${ }^{2}$ Les récits drolatiques concernent particulièrement Le Roman de Renart et les fabliaux qualifiés de littérature de la «risée » ou littérature du gros rire. Ces textes sont définis comme «des contes à rire », comme «de bonnes histoires à servir après le repas», et racontés par «anvoiseüre » (plaisanterie), «por deliter» (pour s'amuser) lors des fêtes et des veillées, comme le déclare, dans le prologue, l'auteur de la branche IV, Ysengrin dans le puits :

«Or me convient tel chose dire

Dont je vos puisse fere rire.

Maintenant, il faut que je vous raconte

Une histoire qui vous divertisse. » Cf : Michel Stanesco, Lire le Moyen Âge, Paris, Dunod, 1998, p. 86.

${ }^{3}$ Ancien nom du renard en ancien français, langue en vigueur au début de l'ère carolingienne (IXe siècle).

${ }^{4}$ L'immortalité de Renart tire sa vitalité et sa pérennisation (la vitalité et la pérennisation de l'immortalité ?!?) dans la mort feinte, ruse qui consiste, de la part du décepteur, à adopter pendant un temps plus ou moins limité l'attitude d'un animal mort : l'animal s'allonge sur le dos et reste vigoureusement immobile en retenant son souffle ; les pattes en l'air, les yeux fermés, les babines retroussées sur les dents. Cette technique est utilisée dans les aventures de chasse et lors des duels
} 
Le texte renardien met admirablement en scène, en effet, un héros rusé et, perpétuellement, en fuite ; ce qui confère au Roman de Renart, ${ }^{5}$ une poïesis, une œuvre en train de se faire, toujours se faisant dans la relance perpétuelle des aventures cocasses et époustouflantes du goupil. La saga renardienne développe une problématique dans laquelle s'inscrit l'esprit de cette réflexion: Renart, personnage des frontières. Les conteurs, en jetant délibérément dans l'arène narrative un personnage famélique, instable, insaisissable et foncièrement madré, soulèvent le problème de l'identité ${ }^{6}$ de celui-ci : qui est Renart ? Dans quelle mesure le personnage éponyme participe-t-il du fondement de l'ensemble des récits ? Comment assume-t-il cette spécificité ? Peut-on définitivement freiner l'élan de ce héros-décepteur? Dans une trajectoire analytique bipartite, le jeu du personnage (jeu de la narration) sera saisi, et l'on s'interrogera sur les enjeux d'une telle mobilité incessante.

\section{L'ethos du personnage}

Le personnage central de «Renart», figure emblématique au premier degré d'une société féodale régie par l'âpreté des relations de force, est un baron, mais qui persécute sans vergogne les autres. Devenu une sorte de figure légendaire du fripon maléfique, Renart agit en tout puissant et n'obéit qu'à ses besoins immédiats (la faim, la convoitise) ou au désir pervers de faire le mal pour le mal. Il se place au-dessus des lois et des codes de l'honneur, et s'illustre ainsi, en véritable brigand, investissant tous les univers de l'œuvre. Par ses agissements, le personnage est en permanence dans une posture de fugitif.

\section{La caractérisation du personnage : un héros en perpétuelle fuite}

La fuite constitue l'un des fondements de l'existence du personnage-levier, Renart. Elle est intimement liée à la ruse qui ouvre ses multiples possibilités. L'ethos du goupil se construit dans le défilé de la narration et trouve sa plénitude avec la réitération de ses échappées, marqué par le déploiement incessant de nouvelles branches ${ }^{7}$. Les récits suivent leurs cours et trouvent leur acmé dramatique toutes les fois que Renart prend la poudre d'escampette ou pique des éperons pour échapper à ses poursuivants.

Le châtelain de Maupertuis court pour éviter le gibet, image fixe et horizon d'attente le menaçant sans cesse lors de ses différents jugements; jamais, il ne

judiciaires. Elle n'est pas seulement un moyen de gagner du temps, mais de s'assurer de l'avenir ; elle n'est pas non plus un moyen ponctuel de répondre à un besoin, elle est une façon de (faussement) mourir pour (vraiment) renaître, d'entrer en clandestinité pour mieux régner. Cf. Micheline de Combarieu du Grès, «Faire la mort veille : la ruse de la mort feinte dans Le Roman de Renart », in Prisma VII /2, CESCM, Poitiers, juillet, décembre, 1991, p. 170.

${ }^{5}$ L'édition sur laquelle nous travaillons et dont sont extraites toutes les citations est la suivante : Jean Dufournet et André Méline, Le Roman de Renart, Paris, Garnier-Flammarion, 2 tomes, 2005.

${ }^{6}$ Moessinger Pierre, Le Jeu de l'identité, Paris, PUF, 2000, p. 179.

${ }^{7}$ Pour désigner les récits de la matière renardienne, on a recouru dès le Moyen Âge à un terme nouveau, celui de branche, qui apparaît pour la première fois, dans son sens littéraire de « conte », terme dont l'attestation la plus ancienne se trouverait, selon Noboru Harano, dans la branche IV (vv. 18-21), juste avant l'aventure du puits. 
s'arrête ou ne se repose durablement. La course est son principe de vie; une démarche qui informe toute l'écriture, faisant reposer la variation de ses aventures sur un même thème : la quête alimentaire.

Épopée de la ruse, mais aussi et surtout épopée de la faim ${ }^{8}$, Le Roman de Renart jette dans l'arène un héros au ventre toujours creux, arpentant infiniment les différents espaces et lieux de l'œuvre. La faim accablante, redoutable, se présente comme un problème de survie individuelle dans le tissu narratif de l'œuvre : chacun à son tour semble connaître la faim, aussi bien le puissant que le faible.

La branche IV, Ysengrin dans le puits, montre Renart tourmenté par la faim se diriger à vive allure vers l'abbaye des moines :

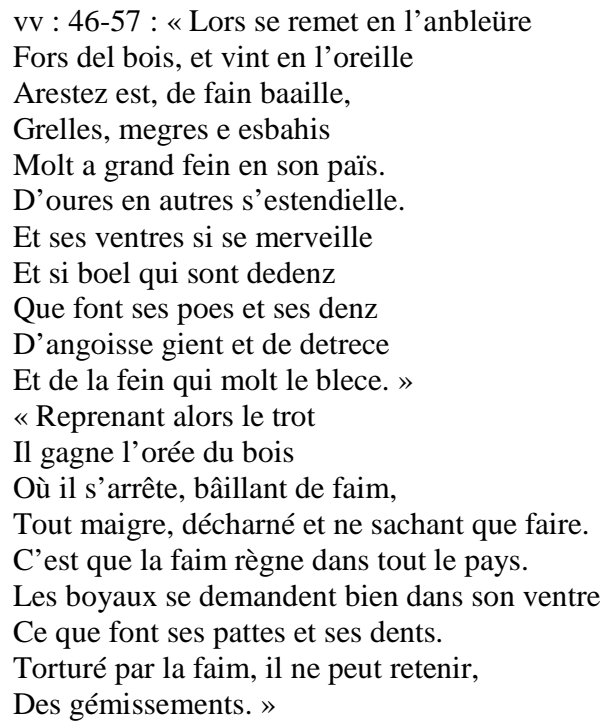

Ses quêtes de nourriture se réalisent sur fond de danger. D'ailleurs, il en est conscient. Subvenir aux besoins alimentaires de la famille est un impératif absolu. Le devoir parental est donc si fort que parfois, Renart, en dépit des dangers représentés par ses expéditions, malgré les périls auxquels il s'expose, s'entête à accéder à ses quêtes. Le pari est clair : "Qui chaut tout est en aventure / Qui ne risque rien n'a rien. », (branche IV, v. 106).

De ce fait, le goupil est un personnage perpétuellement sur le qui-vive. Ses rapports avec les hommes procèdent d'un système de convoitise et d'opposition : l'animal convoite la viande (les poules en particulier) et l'homme sa peau. Dès lors, le goupil adopte le trait de comportement caractéristique de l'animal traqué : la fuite est une réaction instinctive face au danger et à la menace que représente l'homme, d'où l'utilisation par les conteurs de la tournure expressive employée

\footnotetext{
${ }^{8}$ Michelle Augier, «Le Thème de la faim dans les premières branches du Roman de Renart ", in : Mélanges Jeanne Lods du Moyen Âge au XXe siècle, Paris, École Normale Supérieure (École Normale des jeunes filles), 1978, tome 1, pp. 40-48.
} 
cinq fois dans la branche $\mathrm{II}^{9}$, aux vers $351 ; 465 ; 647 ; 783 ; 834:$ «.. fuiant s'en va / prend la fuite», ou «s'en va fuiant / prend la fuite. » Le personnage en fuite «ne suit jamais un chemin rectiligne ${ }^{10}$, mais bien une trajectoire en zigzag qui lui permet de semer, « desvoier / d'égarer» les chiens.

\section{2-Les itinéraires du héros : un espace multiforme}

Dans le récit renardien, l'intrigue se constitue en un double mouvement qui dessine et délimite l'espace. La narration est sujette aux subtilités des gestes et actions de Renart, elle s'oppose à la réalité du personnage, comme le souligne Michel Pastoureau :

«Le renard, en effet, se tient plus volontiers en surface que sous terre. Tapi dans un fourré, couché dans un buisson, caché dans une cavité naturelle ou de construction humaine, il se repose, dort, écoute, observe, sent, guette ses proies. Le terrier n'est pour lui qu'un abri provisoire d'inaction. $\gg^{11}$

La mobilité spatiale de l'animal-héros est systématique, cela confère aux récits une fragmentation narrative rendant dynamique l'espace renardien. Dans la branche III, Les poissons volés, Renart passe « entre le bois et la rivière », (v. 12), puis « s'est couchier lez une haie / s'est couché le long d'une haie », (v. 20). Dans la branche IV, Ysengrin dans le puits, «s'en va fors del bois / s'en va pour sortir du bois », (v. 47).

Ailleurs, il rencontre Tibert et «Andui s'en tournent une sente / chemine le long d'un sentier», (v. 101). Pour rejoindre Chanteclerc le coq, le goupil «s'en vint traiant à une vile, la vile seoit en un bos / se dirigea vers une ferme située dans un bois », (vv. 26-27). Dans la branche II, Renart et Tibert, pour se venger du chat, Renart s'est mis au bord de l'ornière qui marquait la limite entre le bois et un étroit sentier : « Si a choisi près de l'ornière, entre le bois et la carere / il l'aperçut près de l'ornière entre le bois et le chemin charretier. », (vv. 725-726). La branche II d, Renart et Hersent, montre le goupil qui, avant de se retrouver dans la demeure du loup, passe à travers un bois, «Renars vint par un bois fendant / Renart se met en route coupant à travers le bois », (v. 1027), poursuit son chemin jusqu'à une haie « tant que il vint en une haie / si bien qu'il s'y arriva », (v. 1030).

Le héros dans Les vêpres de Tibert, fait un long détour. De son château il passe, en effet, le «bois de Veneroi », coupe ensuite par la lande avant de suivre une «voie » qui débouche sur un poulailler où il est aperçu par les lévriers, (vv. 16-18). La branche IX, Brun, Renart et Liétart, présente le goupil en chasse depuis le matin dans la forêt, non loin d'un chemin « en un bois après del chemin / dans le bois au-delà du chemin » (v. 442). Ayant perçu des aboiements de chiens, il se

\footnotetext{
${ }^{9}$ Cette branche, la plus ancienne, est une composition par enfilage, un récit à tiroirs. Il y a, en effet,

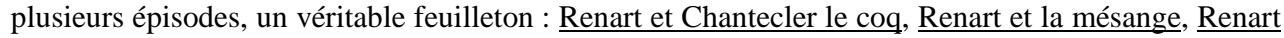
et Tibert le chat, Renart et Tiécelin le corbeau et Renart et Hersent la louve.

${ }^{10}$ Cette technique est connue des bestiaires. Cf : Bestiaires du Moyen Âge, Textes de Pierre de Beauvais, Guillaume Le Clerc, Richard de Fournival, Bruneto Latini, Corbechon, mis en français moderne par Gabriel Bianciotto, Paris, Ed. Stock, Moyen Âge, 1980, p. 37.

${ }^{11}$ Michel Pastoureau, «Introduction à l'étude du goupil », in Prisma, VII / 2, CESCMS, Poitiers, juillet, décembre, 1991, pp. 431-446.
} 
cache dans le creux d'un chêne, pour les laisser passer. Puis, quittant le bois pour les champs, il court à vive allure : « del bois ist, a l'essart va droit / quittant le bois, il fila directement à l'essart » (v. 463).

De l'intérieur de son château, espace privé, le décepteur investit les lieux clos des essarts des vilains ou des moines à la recherche de géliniers dans le but de capturer des chapons ou des gélines :

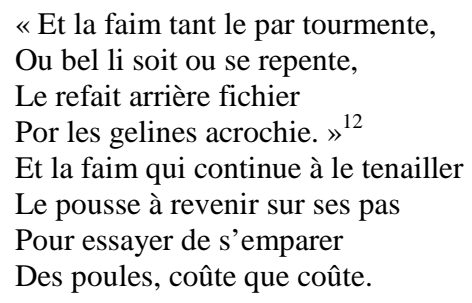

Ces endroits deviennent pour Renart des univers professionnels, sociaux qui lui procurent de quoi subvenir à ses besoins et à ceux de sa famille. Perpétuellement en quête, il assemble les aventures entre elles, non pas tant comme actant récurrent, mais surtout parce qu'il est dans le besoin, et donc constamment en déplacement, justifiant ainsi, le proverbe médiéval : «Besoing fait vielle troter. / Le besoin fait même trotter les vieilles. » Renart est par essence un personnage cinétique : le mouvement est son mode d'existence, de survie. Ses trajets de quête de nourriture et ses fuites répétées pour rejoindre Maupertuis, son château, couvrent un espace multiforme.

Le personnage animal, à l'extérieur, ne s'exerce jamais, d'ailleurs, sur un espace complètement ouvert ou totalement fermé. Il est à la frontière de la forêt et des hameaux, se révélant, en effet, comme le héros des clairières et des sentiers. Le goupil affamé quitte sa «tesnière » (tanière) en direction d'une ferme, opère, et, pourchassé par les vilains et leurs mâtins, revient bredouille ou chargé de butin dans son terrier.

Ainsi, le goupil fugitif ne permet à aucun conteur de donner un tableau macroscopique des lieux; d'où la description topographique se réduit à la simple nomination ou au simple inventaire des espaces internes (terrier, tanière, pertuis) et externes (fermes, clôtures, murs). L'on peut convenir avec Rosanna Brusegan sur la représentation spatiale dans les fabliaux : «Le caractère expéditif des actions des personnages et le rythme accéléré de la narration sont le résultat de la pauvreté de la description et de la présence des systèmes alternatifs de représentation spatiale. $»^{13}$

Les lieux ne sont perceptibles qu'à travers le parcours des personnages. La trajectoire de Renart est rigoureusement fonctionnelle. La fonctionnalité textuelle prend à témoin l'espace qui signifie : le terrier correspond au repos, à l'inaction, à la sécurité, alors que le sentier, la clairière, la ferme, les murs des abbayes, correspondent à l'action et, partant, au danger.

\footnotetext{
${ }^{12}$ Branche IV, Ysengrin dans le puits, vv. 117-120.

${ }^{13}$ Rosanna Brusegan, «La Représentation de l'espace dans les fabliaux. Frontières intérieures, fenêtres. », in Reinardus, Vol. IV, 1991, p. 53.
} 
Si l'on considère le goupil humanisé, il devient Renart le baron. La métamorphose illusoire ${ }^{14}$, par le jeu de l'écriture influence l'espace : la localisation a une topographie humaine. Ce qui installe l'univers renardien dans les isotopies humaine et animale. Ainsi, par opposition à la «tesnière », c'est le terme « palès » qui est le plus souvent utilisé au début des récits. Tels sont les cas des branches Va, (v. 299) ; I, (v. 18) ; VI, (v. 17) et XI, (v. 1754) ${ }^{15}$.

Toute l'intrigue se déroule dans une instabilité, et Renart est généreusement emporté par les flots de cette instabilité. Il est à la frontière, à la lisière des espaces habités et inhabités. Cette configuration de l'univers renardien dégage toute une symbolique à saisir et à comprendre.

\section{Renart entre « renardie » et « diabolie »}

Le personnage de Renart et sa caractéristique, la ruse qui tire son nom de lui, la renardie, s'inscrivent dans cette double perspective du masque et du langage, du langage comme masque par excellence, au point que le personnage du goupil va devenir progressivement, dans la littérature moralisante de ce temps, une figure allégorique, celle du mensonge et de l'imposture, de l'hypocrisie, que les textes appellent désormais renardie. Renart est un maître du langage, mais qui l'utilise pour se tirer des impasses et aussi, quelquefois, détruire son protagoniste. Ce souspoint de l'analyse montrera, d'une part, l'identité fluctuante du héros qui oscille entre renardie et diabolie, et, d'autre part, les enjeux qui en découlent.

\section{La Renardie : une plate-forme de ruses}

L'univers du Roman de Renart est, a priori, celui de la renardie et non de la diabolie $^{16}$. Les premières branches mettent, en effet, en scène un personnage menacé par le gibet qui, cependant, déjoue cette hart, par son $\operatorname{art}^{17}$. À ce sujet, Jean Dufournet, préfaçant l'œuvre d'Élisabeth Charbonnier, relève admirablement la dimension du personnage :

«Les Clercs du Moyen Âge ont découvert en son nom 'Renart', particulièrement au suffixe 'art' la $3^{\mathrm{e}}$ personne du singulier de l'indicatif présent du verbe 'ardre', «brûler » : Renart, c'est celui que brûle le désir qui le pousse toujours vers de nouvelles quêtes. Mais, c'est aussi, l' 'art', la technique, l'artifice, la ruse, l'art magique qui le fait constamment jouer avec la 'hart', la

\footnotetext{
${ }^{14}$ Expression de Roger Bellon constituant le titre de la première partie de sa Thèse de Doctorat d'État: Diversité et unité dans Le Roman de Renart, soutenue le 14 mars 1992 à l'Université de Lyon II. Il appelle "métamorphose illusoire», "La fantaisie des personnages, la double appartenance au monde animal, et au monde humain... Ni vraiment animaux ni vraiment hommes, ils sont 'autres', vivent en marge du monde animal et du monde humain, tout en ayant des attributs caractéristiques de l'un et l'autre de ces deux mondes : on peut parler d'un 'entre-deux' qui est finalement un ailleurs. », p. 29.

${ }^{15}$ Les branches Va, La Cour de Noble ; I, Le jugement de Renart ; VI, Le moniage de Renart ; XI, Renart Empereur.

${ }^{16}$ Sur la distinction entre « Renardie » et « Diabolie », cf Claude Reichler, La Diabolie, la séduction, la renardie, l'écriture, Paris, Minuit, «Critiques », 1979, pp. 77-149.

${ }^{17}$ Sur l'homophonie entre hart et art, voir l'article de Roger Dragonetti, « Renart est mort, Renart est vif, Renart règne », dans La Musique et les lettres : études de littérature médiévale, Genève, Droz, «Publications romanes et françaises », 1996, pp. 419-434.
} 
corde de la pendaison, avec la mort dont il ne cesse de triompher grâce à ses nombreuses ruses. $»^{18}$

Renart se définit par la ruse. En témoignent ces branches :

branche VII, La confession de Renart, vv. 70-74 :

« Je vos dirai ja sans mentir

Qui a fet tante trecherie

Et qui tant home a deçoü

Que par engin que par vertu.»

Je vais vous raconter exactement

La vie de Renart le goupil

Qui a commis tant de tromperies

Et s'est joué de tant d'hommes

Par sa ruse aussi bienque par sa force.

Branche VIII, Le pèlerinage de Renart, vv. 78-79:

« Dist li vilein : « Renart, ne hoigne!

Tu sez tant de guile et de fart. »

-Renart, répliqua le vilain, ne raconte pas d'histoires !

Tu connais tant de ruses et de mensonges.

Branche IX, Brun, Renart et Liétart, vv. 5-6 :

«Afere une novele branche

De Renart qui tant sout de ganche. »

A composer un nouvel épisode des aventures

De Renart, le trompeur aux milles tours.

Renart appartient tout d'abord à une espèce animale qui possède au Moyen Âge une valeur symbolique. Capable, selon les naturalistes, de simuler la mort afin de capturer des oiseaux, le personnage représente avant tout la ruse, tout le lexique médiéval de la ruse le caractérise inextricablement : guile ou art (ruse), barat, bole ou boide (tromperie), tricherie ou engin (tricherie), conchïement (moquerie), lecherie (perfidie). La finalité de cette ruse et des bons tours du personnage s'inscrivent dans le jeu de l'œuvre: «fere rire ». Le châtelain de Maupertuis n'incarne pas alors le mal, mais se révèle comme le Maître ès ruse dont la compétence dans ce domaine trouve pompeusement son expression condensée dans le mot «renardie $»^{19}$ (le règne de la Renardie), néologisme médiéval. En effet, protéiforme, capable de changer de ton, de voix, de langue, de rôle et même d'apparence, Renart introduit un peu de fantaisie dans un monde relativement morne, attirant vers lui les bonnes grâces du lecteur /spectateur.

Le personnage respecte scrupuleusement les valeurs sacro-saintes de la société médiévale. À ce sujet, Jean Batany a une bonne opinion de lui : «La renardie a pour point de départ le respect des normes sociales: loyauté envers les pairs et bonté envers les faibles. ${ }^{20}$ Le goupil s'illustre avec éclat lors des quêtes de

\footnotetext{
${ }^{18}$ Elisabeth Charbonnier, Le Roman de Renart, Paris, LGF, 1987, pp. 6-7.

${ }^{19} \mathrm{Ce}$ vocable se manifeste par «le faux semblant, le mensonge ou la cautèle, le masque qui sont autant de manifestations d'une confuse identité personnelle. », Jean-Pierre Vernant et Marcel Détienne, La Ruse, Paris, UGE, 10 / 18, 1977, p. 7.

${ }^{20}$ Jean Batany, «La Fontaine et la tradition médiévale. Renardie et Asnerie », in EUROPE, $\mathrm{N}^{\circ} 515$, mars 1972, pp. 62-73.
} 
nourriture. Les récits de ce type fonctionnent à la lumière de la métamorphose illusoire alternative ${ }^{21}$, qui présente le personnage à la frontière de l'animal et de l'humain, entre le zoomorphisme et l'anthropomorphisme. Le décepteur est plaisant et sa ruse est plébiscitée. Il est le porte-parole des petites gens écrasées par une noblesse prédatrice, faisant ainsi de lui le symbole du vassal que ses conditions de vie réduisent à des expédients rusés, puisqu'il ne peut compter sur sa force.

Le héros-trickster du fait de son statut textuel et social qui, même s'il est un archétype de la matière renardienne, demeure aussi un véritable vecteur d'une idéologie du groupe, notamment d'une classe de la société médiévale. Le personnage est vu de l'extérieur comme un Robin des bois, et a une conscience morale. Le héros a profité de son expérience et s'est appliqué à lui-même la maxime exprimée par La Fontaine : «Si tu veux qu'on t'épargne, épargne aussi les autres. »

\section{La Diabolie : une rhétorique du mal}

Dans les récits où la parole passe au premier plan ${ }^{22}$, Renart n'est plus le personnage qui fait preuve de renardie, mais plutôt celui dont le mal devient l'épicentre de ses actions. Combattant avec l'arme langagière, c'est dans le domaine verbal que le héros simule et dissimule ses véritables intentions agressives et meurtrières sous de «blanches paroles / paroles trompeuses ». ${ }^{23}$

Renart adopte, ainsi, une autre attitude : il se dévoie, remettant en cause le statut de personnage éponyme en fuite de son roman. Il représente alors le Mal sous toutes ses formes, prototype de l'Homme démasqué de ses bienséances: «Renart est l'incarnation du Mal [...], les vertus cessent d'exister et sont partout remplacées par les vices. » ${ }^{24}$ C'est le règne de la Diabolie, «mécanisme qui confisque l'être au profit du paraître », selon Zigui Koléa Paulin. ${ }^{25}$

Les conteurs ne voient en lui que le symbole odieux de l'hypocrisie, du mensonge et de la trahison. Renart est à la frontière du Bien et du Mal. Par moments plaisant, mais aussi inquiétant, farceur ici et cynique là, habile comédien ou reflet de la perfidie, ce trompeur exceptionnel et séduisant entame le processus de construction d'une identité fluctuante, instable et d'un discours qui s'achemine vers la connaissance de l'Homme.

Drôle et sinistre, polymorphe et complexe, et fascinant surtout par sa capacité à défier ouvertement l'ordre et de s'inscrire dans des aventures souvent stériles, le

\footnotetext{
${ }^{21}$ Konan Yao Lambert, «La Métamorphose illusoire ou la représentation mutante du personnage dans Le Roman de Renart», in Lettres d'Ivoire, Revue Scientifique de Littératures, Langues et Sciences Humaines, $\mathrm{n}^{\circ}$ 03, Université de Bouaké, Côte d'Ivoire, 2007, p. 232.

${ }^{22}$ Renart et Chantecler le coq, Renart et la mésange, Renart et Tibert le chat, Renart et Tiécelin le corbeau sont les épisodes de la branche II, écrite par Pierre de Saint Cloud.

${ }^{23}$ Branche IX, Brun, Renart et Liétart, vers 965.

${ }^{24}$ Härmä Suomela Elina, «Évolutions et Mutations dans le second cycle de Renart», in L'Information littéraire, $\mathrm{n}^{\circ} 10,1998$, pp. 45-59.

${ }^{25}$ Zigui Koléa Paulin, Les Contes à rire de la France médiévale, Le Roman de Renart et les contes d'animaux de l'Afrique de l'Ouest: Étude de Morphologie et de Physiologie comparées, Types, Structures, Idéologies. Thèse de Doctorat d'État, Université François Rabelais de Tours, 1995, p. 527.
} 
décepteur entreprend une démarche en parallèle, une sorte de contrepoint, au monde des règles et des bienséances. Caractérisé par son ambiguïté, il fait la parade de sa misère, mais aussi de l'attrait suscité par le côté obscur de l'Homme qu'il évoque et représente : «Dans la collection primitive, Renart apparaît avec sa verve gouailleuse, non comme un redresseur de torts, mais comme un juge malicieux qui dénonce les ridicules de l'Homme ${ }^{26}$. L'œuvre, saisie dans sa profondeur, raconte la nature primaire des hommes, leur avidité, leur sottise et la suprématie de la loi du plus fort. Tout cela s'incarne dans le personnage-vedette, figure emblématique du fripon maléfique.

\section{Le comique renardien : une satire sociale}

Le personnage de Renart est une interpellation à la conscience humaine, comme le soulignait Kouacou Jacques Raymond Koffi dans une étude, Le Roman de Renart: une interpellation à la conscience humaine. ${ }^{27}$ Les récits mettent en scène ce personnage sériel pour symboliser l'Homme confronté à la sempiternelle lutte entre le Bien et le Mal.

Le héros éponyme donne une coloration hybride à l'œuvre qui ressemble tantôt à une fable, tantôt à une épopée (récit d'aventures) amusante. Cet hybridisme tire sa vitalité de la satire des branches, faisant de ce joyau littéraire médiéval une œuvre caustique dénonçant les vicissitudes humaines, par le biais du rire qui se dégage des actions d'éclat du héros. L'œuvre s'inscrit dans les contes à rire de la France médiévale; le rire acquiert toute sa dimension, il a un rôle aussi bien narratif que thématique ou idéologique.

Hérité du latin populaire «ridëre ${ }^{28}$, qui désigne soit la manifestation physique du rire (forme transitive), soit sa réalisation intellectuelle dans le sens de «se moquer de» (forme intransitive), le rire est étroitement lié au comique, au point qu'ils se confondent. Le comique est souvent indirectement désigné par le rire, l'humour, la parodie. Le rire et le comique présentent, souvent, une confusion au point que le premier est employé pour désigner le second. Ils sont envisagés comme des quasi-synonymes. C'est à juste titre qu'Henri Bergson décrit le rire (qu'il identifie au comique) comme « un certain geste social, qui souligne et réprime une certaine distraction spéciale des hommes et des événements. ${ }^{29} \mathrm{Il}$ les considère comme une sanction sociale visant à réprimer tout écart à la norme. Le rire devient donc une arme : c'est la portée subversive du comique.

Le Roman de Renart écrase la frontière entre normalité et transgression, et livre un texte satirique fonctionnant sur le régime du comique. L'œuvre comporte, en effet, une multitude de procédés $\operatorname{comiques}^{30}$ (de situation, de mots et de

\footnotetext{
${ }^{26}$ Robert Bossuat, Dictionnaire des Lettres, Le Moyen Âge, Paris, Bordas, 1964, p. 653.

${ }^{27}$ Kouacou Jacques Raymond Koffi, « Le Roman de Renart: une interpellation à la conscience humaine », in Lettres d'Ivoire, Revue Scientifique de Littératures, Langues et Sciences Humaines, Université de Bouaké, Côte d'Ivoire, n 009, 2010, pp. 132-142.

${ }^{28}$ Algirdas Julien Greimas, Dictionnaire de l'Ancien Français, Paris, 2001 et Dictionnaire historique de la langue française, Alain Rey (dir.), Paris, Robert, 1992.

${ }^{29}$ Henri Bergson, Le Rire : Essai sur la signification du comique, Paris, PUF, 1999. p. 67.

${ }^{30}$ Armelle Leclercq, «Renart ou le rire rebelle », in Études littéraires, vol. 38, 2007, pp. 87-100.
} 
gestes) : certains sont axés sur un sujet risible (thèmes du bas corporel et du cocuage), d'autres sur un emploi décalé du langage (burlesque, héroï-comique, mélange de langues), d'autres encore sur un usage ludique de la littérature dite « sérieuse » (parodie de la chanson de geste, du roman courtois).

Les frontières entre le sérieux et le comique sont ainsi poreuses. Le ton des épisodes est très varié, sérieux aussi bien que burlesque ou ironique, à l'image de son personnage à la limite de ces frontières littéraires. Le spectateur ou le lecteur rit des défauts des hommes (la naïveté d'Ysengrin), mais aussi du fonctionnement de toute la société $e^{31}$.

Un fait capital est toutefois bien attesté : au Moyen Âge, le rire est libérateur. De qui, de quoi rit-on? La pertinence des réponses à ces questions passe par une migration au cœur des structures sociales et des mentalités collectives d'une société qui se contemple dans un miroir, et aperçoit ses ridicules ${ }^{32}$. Le rire renardien s'inscrit dans la perspective d'un rapport de dilemme avec une société qui ne répond pas à ses expectatives, parce qu'elle a remplacé l'être par le paraitre.

Le discours littéraire comique est alors un prétexte et un réflexe médiévaux pour l'actualisation littéraire de cette figure archétypale (Renart). À ce niveau, l'Homme laisse entrevoir ses besoins fondamentaux, ses faiblesses et ses contradictions. Le comique représente l'espace pour l'énonciation de l'autre visage de la réalité, voire son côté terrible et violent. La finalité sans fin de ce genre satirique devient ainsi un instrument utile pour se défendre de la peur, pour difformer certains paramètres sociaux et en rendre possible un affranchissement du moins provisoire.

La perspective comique est le filtre à travers lequel sont empruntés au monde effectif les éléments qui intéressent pour mieux montrer la réalité dans sa profondeur. La typification, la stylisation, les traits généraux et les plus représentatifs constituent une voie d'écart qui rend possible cette démarche d'observation et d'approfondissement. Peindre l'humanité dans sa médiocrité réelle pour en dénoncer les défauts, en cela ce mode narratif (le comique) gagne en intérêt.

Renart fascine parce qu'il amplifie quelque chose de nous-mêmes, il sert d'exutoire à nos zones d'ombres. Le personnage révèle ainsi la toute puissante sauvagerie de nos égos infantiles, nos désirs primaires de possession; en somme tout ce qu'une éducation bien pesée devrait civiliser.

\section{Conclusion}

L'étude a montré que ce personnage à griffes est, à tous points de vue, animé. Tour à tour teinturier, médecin, moine, jongleur, faussaire, régent, baron, bref, personnage protéiforme, Renart est à la frontière de tous les mondes et en marge du monde dans lequel il vit. Le héros repousse les frontières et fait vaciller les

\footnotetext{
${ }^{31}$ Jacques Le Goff, «Le Rire dans la société médiévale », in Un Autre Moyen Âge, Paris, Quarto Gallimard, 1999, p. 1343.

${ }^{32}$ Philippe Ménard, «Le Rire et le sourire au Moyen Âge dans la littérature et dans les arts : essai de problématique », in Le Rire au Moyen Âge dans la littérature et dans les arts, éd. T. Boucher, H. Charpentier, Bordeaux, Presses Universitaires de Bordeaux, 1990, pp. 9-30.
} 
bornes qui contraindraient ses déplacements. Ce qui fait dire à Aurélie Barre qu' «il ne peut être contenu par une image-prison. » ${ }^{33}$

Renart, tel le phénix, renaît toujours de sa fausse mort. Se tenant à la lisière de la vie et de la mort, du Bien et du Mal, le décepteur résume la condition humaine. $\hat{A}$ la fois personnage d'un cycle, personnage dans une suite / continuation ${ }^{34}$, dans une transposition / réécriture ${ }^{35}$ et dans une adaptation cinématographique ${ }^{36}$, le héros est entré dans l'histoire de l'humanité, car devenu personnage-migrant ${ }^{37}$.

\section{RÉFÉRENCES BIBLIOGRAPHIQUES}

\section{Le corpus}

Dufournet Jean et Meline André (2005), Le Roman de Renart, Paris, GarnierFlammarion, 2 tomes.

\section{Les autres textes consultés}

AUGIER Michelle (1978), "Le Thème de la faim dans les premières branches du Roman de Renart», in Mélanges Jeanne Lods du Moyen Âge au XXe siècle, Paris, École Normale Supérieure (École Normale des jeunes filles), tome 1, pp. 40-48.

BATANY Jean (mars 1972), «La Fontaine et la tradition médiévale. Renardie et Asnerie », in EUROPE, $\mathrm{N}^{\circ} 515$, pp. 62-73.

BARRE Aurélie (2013), «Renart personnage animé », in Textimage, Varia 3, Revue d'étude du dialogue Texte-image, pp. 2-17.

BELLON Roger (1999), Diversité et unité dans Le Roman de Renart, Thèse de Doctorat d'Etat, Université de Lyon II.

\footnotetext{
${ }^{33}$ Aurélie Barre, «Renart personnage animé », in Textimage, Varia 3, Revue d'étude du dialogue Texte-image, 2013, pp. 2-17.

${ }^{34}$ Rutebeuf, Renart le bestourné, en 1261, Jacquemart Gielée et le Clerc de Troyes rédigent respectivement Renart le nouvel et Renart le contrefait entre la fin du XIIIe et du premier tiers du XIVe siècle.

35 «L'intérêt de Renart, et celui des fabliaux, c'est que la tradition les a perpétués : Boccace, Rabelais, Molière et La Fontaine en témoignent. », Robert Sabatier, La Poésie du Moyen Âge, Paris, Albin Michel, 1975, pp. 246-247.

${ }^{36}$ Le projet d'adaptation au cinéma du Roman de Renart date de 1929 : Ladislas Starewitch conçoit et fabrique des marionnettes, Irène Starewitch rédige un scénario. Le film sort le 10 avril 1941. Cf, Olivier Lepâtre «Un épigone filmé : Le Roman de Renart de Ladislas et Irène Starewitch », dans Éditer, traduire ou adapter les textes médiévaux, Actes du Colloque international des 11 et 12 décembre 2008, Université Jean Moulin, Lyon 3, C. E. D. I. C, Centre Jean Prévost, pp. 313-327.

${ }^{37}$ Umberto Eco, dans Sur quelques fonctions de la littérature, explique que des personnages comme Le Petit Chaperon Rouge, d'Artagnan ou Alice ont une existence en dehors des «partitions originales» et existent même pour qui n'a jamais lu le texte de départ: "ils sont devenus collectivement vrais parce que la communauté a fait, sur eux des investissements passionnels. », in Mensuel, n³92, 2000, pp. 58-64.
} 
BERGSON Henri (1999), Le Rire : Essai sur la signification du comique, Paris, PUF.

Bianciotto Gabriel (1980), Bestiaires du Moyen Âge, Textes de Pierre de Beauvais, Guillaume Le Clerc, Richard de Fournival, Bruneto Latini et Corbechon, Paris, Ed. Stock, Moyen Âge.

Bossuat Robert (1964), Dictionnaire des Lettres, Le Moyen Âge, Paris, Bordas.

BRUSEGAN Rosanna (1991), «La Représentation de l'espace dans les fabliaux. Frontières intérieures, fenêtres. », in Reinardus, Vol. IV, p. 53.

CHARBOnNIER Elisabeth (1987), Le Roman de Renart, Paris, LGF.

COMBARIEU du Grès Micheline de (1991), «Faire la mort veille : la ruse de la mort feinte dans Le Roman de Renart », inPrisma VII /2, CESCM, Poitiers, juillet, décembre, p. 170.

DETIENNE Marcel et VERnANT Jean-Pierre (1977), La Ruse, Paris, UGE, 10 / 18.

DRAGONETTI Roger (1996), « Renart est mort, Renart est vif, Renart règne », in La Musique et les lettres : études de littérature médiévale, Genève, Droz, « Publications romanes et françaises », pp. 419-434.

ECO Umberto (2000), «Sur quelques fonctions de la littérature », in Mensuel, n³92, pp. 58-64.

GrEIMAS Algirdas Julien (2001), Dictionnaire de l'Ancien Français, Paris.

KONAN Yao Lambert (2007), « La Métamorphose illusoire ou la représentation mutante du personnage dans Le Roman de Renart », in Lettres d'Ivoire, Revue Scientifique de Littératures, Langues et Sciences Humaines, $\mathrm{N}^{\circ} 03$, Université de Bouaké, Côte d'Ivoire, p. 232.

KoUACOU Jacques Raymond Koffi (2010), « Le Roman de Renart: une interpellation à la conscience humaine", in Lettres d'Ivoire, Revue Scientifique de Littératures, Langues et Sciences Humaines, Université de Bouaké, Côte d'Ivoire, $\mathrm{N}^{\circ}$ 009, pp. 132-142.

LECLERCQ Armelle (2007), « Renart ou le rire rebelle », in Études littéraires, vol. 38, pp. 87-100.

LE GoFF Jacques (1999), «Le Rire dans la société médiévale », in Un Autre Moyen Âge, Paris, Quarto Gallimard, p. 1343.

LEPATRE Olivier (2008), «Un épigone filmé : Le Roman de Renart de Ladislas et Irène Starewitch », in Éditer, traduire ou adapter les textes médiévaux, Actes du Colloque international des 11 et 12 décembre 2008, Université Jean Moulin, Lyon 3, C. E. D. I. C, Centre Jean Prévost, pp. 313-327.

MENARD Philippe (1990), «Le Rire et le sourire au Moyen Âge dans la littérature et dans les arts : essai de problématique », in Le Rire au Moyen Âge dans la littérature et dans les arts, éd. T. Boucher, H. Charpentier, Bordeaux, Presses Universitaires de Bordeaux, pp. 9-30.

Moessinger Pierre (2000), Le Jeu de l'identité, Paris, PUF.

PASTOUREAU Michel (1991), «Introduction à l'étude du goupil », in Prisma, VII /

2, CESCMS, Poitiers, juillet, décembre, pp. 431-446.

REICHLER Claude (1979), «La Diabolie, la séduction, la renardie, l'écriture », in, Critiques, Paris, Minuit, pp. 77-149.

REY Alain (1992), Dictionnaire historique de la langue française, Paris, Robert. SABATIER Robert (1975), La Poésie du Moyen Âge, Paris, Albin Michel. 
SUOMELA-HÄRMÄ Elina (1998), «Évolutions et Mutations dans le second cycle de Renart », in L'Information littéraire, $\mathrm{N}^{\circ} 10$, pp. 45-59.

STANESCO Michel (1998), Lire le Moyen Âge, Paris, Dunod.

Zigui Koléa Paulin (1995), Les Contes à rire de la France médiévale, Le Roman de Renart et les contes d'animaux de l'Afrique de l'Ouest: Étude de Morphologie et de Physiologie comparées, Types, Structures, Idéologies. Thèse de Doctorat d'État, Université François Rabelais de Tours. 